\title{
Fetal Hippocampal Development: Analysis by Magnetic Resonance Imaging Volumetry
}

\author{
FRANCOIS DOMINIQUE JACOB, PIOTR A. HABAS, KIO KIM, JAMES CORBETT-DETIG, DUAN XU, COLIN STUDHOLME, \\ AND ORIT A. GLENN
}

\author{
Division of Pediatric Neurology [F.D.J.], University of Alberta, Edmonton, T6G-2J3 Alberta, Canada; Department of Radiology \& \\ Biomedical Imaging [P.H., K.K., J.C.-D., D.X., C.S., O.A.G.], University of California, San Francisco, San Francisco, California 94143
}

\begin{abstract}
The hippocampal formation plays an important role in learning and memory; however, data on its development in utero in humans are limited. This study was performed to evaluate hippocampal development in healthy fetuses using 3D reconstructed MRI. A cohort of 20 healthy pregnant women underwent prenatal MRI at a median GA of 24.9 wk (range, 21.3-31.9 wk); six of the women also had a second fetal MRI performed at a 6-wk interval. Routine 2D ultrafast T2-weighted images were used to reconstruct a $3 \mathrm{D}$ volume image, which was then used to manually segment the right and left hippocampi. Total hippocampal volume was calculated for each subject and compared against GA. There was a linear increase in total hippocampal volume with increasing GA $(p<0.001)$. For subjects scanned twice, there was an increase in hippocampal size on the second fetal MRI ( $p=0.0004)$. This represents the first volumetric study of fetal hippocampal development in vivo. This normative volumetric data will be helpful for future comparison studies of suspected developmental abnormalities of hippocampal structure and function. (Pediatr Res 69: 425-429, 2011)
\end{abstract}

$\mathrm{T}$ he hippocampal formation plays an important role in the cognitive function of learning and memory (1). Development of the hippocampal formation occurs via the progressive infolding of the fetal dentate gyrus, cornus ammonis, subiculum, and parahippocampal gyrus forming the hippocampal fissure (2). Abnormalities of the hippocampal formation have been described in several congenital malformations including agenesis of the corpus callosum, holoprosencephaly, polymicrogyria, and lissencephaly, and in children with epilepsy (3-5). During its development in utero, the hippocampal formation is vulnerable to hypoxic-ischemic insult, stress, undernutrition, and metabolic disturbance (6-8). Injury or impairment of hippocampal development may be a contributor to the neurodevelopmental burden of affected individuals. Indeed, reduction in hippocampal volume has been associated with impaired memory and learning in older preterm children (9-13).

Numerous hippocampal formation volumetric studies have been performed in the adult and pediatric populations and, more recently, in neonates. However, there is a lack of established methodology to segment the hippocampus in the fetal

Received July 13, 2010; accepted November 24, 2010.

Correspondence: Orit A. Glenn, M.D., UCSF Department of Radiology, Neuroradiology Section, 505 Parnassus Avenue, Box 0628, San Francisco, CA 94143-0628; e-mail: Orit.Glenn@ucsf.edu

Supported by grants by the National Institutes of Health K23 NS 52506 [to O.A.G.] and R01 NS 055064 [to C.S.]. population. Furthermore, there is a lack of normative data on hippocampal volumes in living fetuses. There have been a few MRI studies on the hippocampus in the fetal population, although these have been limited to two dimensional assessments in postmortem fetuses or in vivo fetuses $(2,14)$. With recent advances in image processing methods (15-19), 3D segmentation of the fetal hippocampus can now be performed using in utero MR images.

Prenatal MR volumetry of the hippocampal formation may provide additional insight into normal in utero hippocampal development as well as developmental and acquired disorders in which the hippocampus may play an important role. The aims of this study are a) to develop a method for 3D segmentation of the fetal hippocampus based on adaptations of established methodology in the pediatric and adult populations; $b$ ) to apply this methodology to healthy fetuses in the second and third trimesters using 3D reconstructed MRI; and c) to establish normative volumetry data in this population and set a benchmark for future studies.

\section{METHODS}

Subjects. A cohort of 20 healthy pregnant women underwent prenatal MRI without maternal or fetal sedation. All patients had normal detailed ultrasound and normal fetal MR neuroimaging. Twelve women underwent fetal MRI as volunteers for a larger fetal MRI study; six women underwent fetal MRI because of a history of a prior child or fetus with brain abnormality; and two women underwent fetal MRI for a questionable brain abnormality on outside screening ultrasound with a subsequent normal detailed ultrasound. Median GA at the time of fetal MRI was 24.9 wk based on last menstrual period (range, 21.3-31.9 wk). Six patients also underwent a second fetal MRI, which was performed on average $6.1 \mathrm{wk}$ after the first MRI. This study was approved by University of California San Francisco (UCSF) institutional review board and informed consent was obtained from all subjects.

Data acquisition and image processing. MR scanning was performed on 1.5-T magnet (General Electric, Milwaukee, WI) using a torso-phased array coil. The protocol consisted of 3-mm-thick (no skip) interleaved single-shot fast spin echo T2-weighted images (TR 3000-8000 ms, TE $90 \mathrm{~ms}$ ), obtained in the axial, sagittal, and coronal planes. The image stacks were then transferred off-line, and slice intersection motion correction was applied to estimate relative fetal motion between the acquisition of each MR slice using previous described methods $(16,17)$. The motion parameters were then used to reconstruct a geometrically correct 3D volume image on a regular voxel lattice using a previously described approach (17). The reconstructed images had a voxel size of $0.5 \mathrm{~mm} \times 0.5 \mathrm{~mm} \times 0.5 \mathrm{~mm}$.

Hippocampal segmentation. The 3D image volume was viewed and displayed in coronal, axial, and sagittal planes using the manual segmentation tool in the rView software (http://rview.colin-studholme.net) version 9 running on MAC OS; and the right and left hippocampus were then manually segmented. All hippocampal segmentations were performed by one of the 
authors (F.D.J.), who remained blinded to the GA of the fetus. Segmentation was performed initially in the sagittal plane, followed by the coronal plane, and confirmed in the axial plane. For ease of tracing, the coronal sections were oriented perpendicular to the long axis of the hippocampus, which was best achieved by locating the floor of the right anterior lateral ventricle on a sagittal off midline image, and then orienting the coronal plane so that it was perpendicular to the floor of the lateral ventricle. The right hippocampus was always segmented before the left. The anatomical landmarks and boundaries used for segmentation of the hippocampus were based on prior anatomic and embryologic descriptions (20-23) and prior hippocampal formation volumetric studies $(24,25)$. It is important to note that although the term hippocampal formation refers to a constellation of anatomically distinct structures including the subiculum, Ammon's Horn, and the dentate gyrus, these structures are indistinguishable by fetal MRI and thus were sampled as a whole complex. All segmentations were reviewed by a pediatric neuroradiologist (O.A.G.).

Anatomic boundaries used to segment the hippocampal head were as follows: anterior and lateral borders were delineated by the temporal horn of the lateral ventricle, which is best performed in the sagittal and axial plane; the medial border of the hippocampal head was defined by the cerebrospinal fluid of the ambient cistern; the superior border was defined by the cerebrospinal fluid of the uncal recess of the temporal horn of the lateral ventricle, which overlies the head of the hippocampus laterally; whereas the inferior border was demarcated by the gray/white matter junction. Delimitation of the hippocampal head from the amygdala was best achieved in the sagittal plane, as the two structures are separated laterally by the uncal recess of the lateral ventricle. In most cases, this is easily identifiable. However, the more medial portion of the uncal recess is often obliterated by hippocampal digitations fused to the amygdala across the ventricular cavity. In these cases, a straight line from the inferior horn of the lateral ventricle was drawn to the surface of the uncus as described by Watson et al (25). The superior, medial, and inferior borders of the hippocampal head were best segmented using the coronal and sagittal planes.

Anatomic boundaries used to segment the hippocampal body were as follows: inferior, lateral, and superior borders of the body of the hippocampus were similar to the borders used for the head of the hippocampus. The superior aspect of the medial border of the hippocampal body was defined by the cerebrospinal fluid of the ambient cistern. For younger fetuses, the superior border of the body was separated from the adjacent more hypointense germinal matrix by changing the intensity of the image and by using the sagittal plane. The medial border was defined by the cerebrospinal fluid of the uncal cleft. Inferiorly, the subiculum cannot be differentiated from the hippocampus; therefore, the subicular complex was included in the segmentation, similarly to the methodology by Watson et al (25). The subiculum was segmented up to the most medial portion of the hippocampal head or body.

Boundaries of the tail of the hippocampal formation were similar to those used for the body of the hippocampus. Because the fornices are difficult do delineate in younger fetuses, we chose to limit the posteriomedial extent of hippocampal tail segmentation in all subjects based on sagittal plane that intersected both the medial margin of the hippocampal head and the hippocampal tail; only portions of the tail that were lateral to this plane were included in the total hippocampal volume (THV) segmentation.

THV was calculated for each subject from the segmented data as the summation of the right and left hippocampal volumes. Seven scans were also selected randomly and the right and left hippocampi were resegmented by one of the authors (F.D.J.), who was blinded to initial segmentations, 2-4 wk after the initial segmentation to assess intraobserver variability.

Data analysis. Right and left hippocampal volumes were compared using a paired $t$ test, using a significance level of 0.05. Linear regression analysis was performed on THV with increasing GA. THV for male and female fetuses were compared as a fixed effect. For those fetuses with two fetal MR examinations, the rates of increase in THV between the two time points were compared using linear regression model with mixed random effects. Intraclass correlation coefficient was calculated for the repeat hippocampal segmentations.

\section{RESULTS}

Sagittal, coronal, and axial hippocampal images are shown in Figure 1, illustrating segmentations of the left and right hippocampus of a fetus at 28 wk gestation. An axial view of a 3D surface model based on the hippocampal segmentation is depicted in Figure 2. There was a linear increase in THV between 21 and 31 gestational wk with a slope of $0.0477(p<$ $0.0001 ; R^{2}=0.79$; Fig. 3). Mean THV at 21 gestational wk was $0.177 \mathrm{~mL}$, compared with $0.619 \mathrm{~mL}$ at 31 gestational wk (Table 1). Based on the resultant growth curve, THV can be estimated during 21-31 gestational wk as THV $(\mathrm{mL})=$ $(0.0468 \times$ gestational weeks $)-0.8034$. Estimated THV for different GAs are shown in Table 1.

For all subjects who underwent a second fetal MRI, there was an increase in size of the hippocampus on the second fetal MRI. THV increased with age from first to second scan with a slope of 0.0494 ( $p=0.0004$; Fig. 4).

In all subjects, there was no significant difference in left and right hippocampal volumes $(p=0.1)$ or in the slope of growth curve of left and right hippocampal volumes. Average left and right hippocampal volumes were 0.202 and $0.200 \mathrm{~mL}$, respectively. There was no significant difference between THV in female and male fetuses $(p=0.21)$, with mean THV of 0.422 $\mathrm{mL}$ in females and $0.382 \mathrm{~mL}$ in males; however, mean GA was greater for females (26.7 gestational wk) compared with males (24.9 gestational wk). There was no significant difference between the slope of the growth curve for female versus male fetuses. Intraclass correlation coefficient for the repeat segmentations was 0.995 .

\section{DISCUSSION}

In vivo prenatal MRI is a noninvasive technique that enables us to study the development of the brain throughout gestation. Using the slice intersection motion correction algorithm (17), we were able to reconstruct 3D images of the fetal brain. This allowed us to visualize and segment the hippocampus in multiple planes and, for the first time, report on normal hippocampal volumes in vivo. We observed a linear rate of growth of the volume of the hippocampal formation in vivo during the period of 21 to $31 \mathrm{wk}$ gestation. Moreover, even for those fetuses scanned at two separate time-points, there was a linear increase in THV.

MR-derived hippocampal volumetric techniques have demonstrated good validity and reproducibility in adult studies (25-27). However, there have been numerous methodologies used, which has led to discrepancy among research findings (28). We have based our methodology on the most commonly used adult protocols, namely the methodology by Watson $e t$ al. (25) and Jack et al (24). This is possible because by $21 \mathrm{wk}$ gestation, the relationship of the hippocampal sulcus to the surrounding structures is similar to the adult brain (23). However, numerous adaptations were needed to segment the hippocampal formation effectively in the fetus. The fetal brain consistently changes during the gestation, which makes the choice of fixed landmarks difficult. Therefore, we could not orient the coronal section perpendicular to the plane of the Sylvian fissure, such as described by Jack et al(24). To optimally segment the head of the hippocampus, we oriented the coronal plane perpendicular to the plane of the floor of the lateral ventricle. This enabled effective visualization of the head, body, and tail of the hippocampus and their relationship to neighboring structures such as the amygdala and thus allowed us to apply the methodologies of Watson et al. and Jack et al. 
A

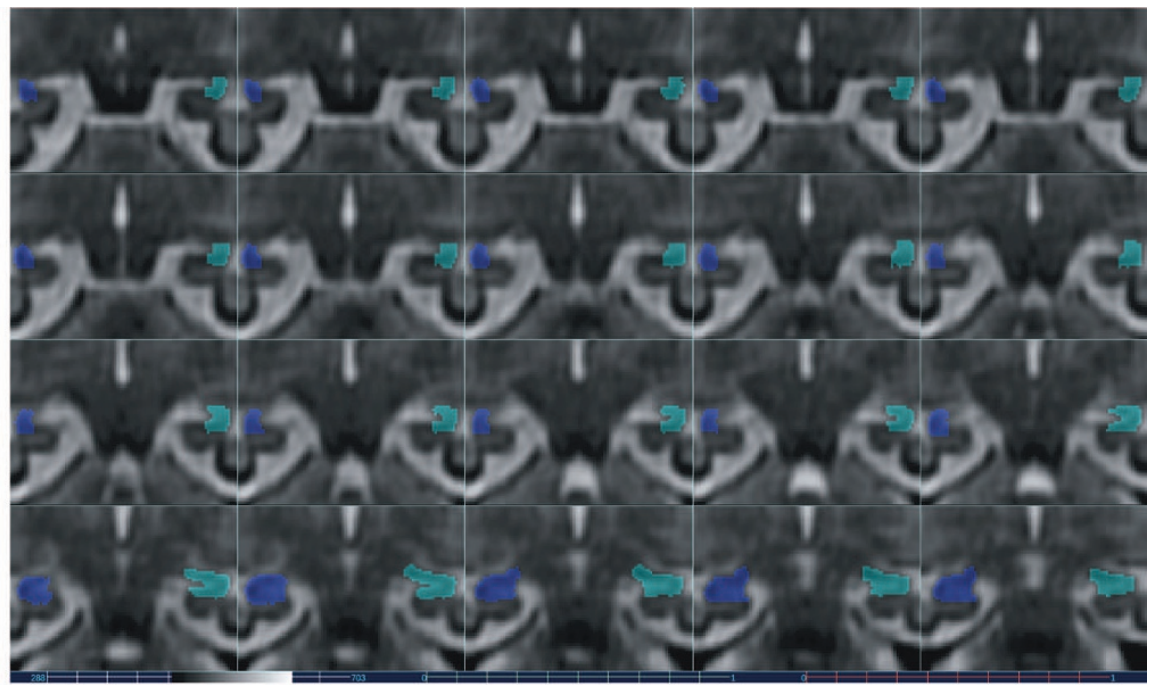

B

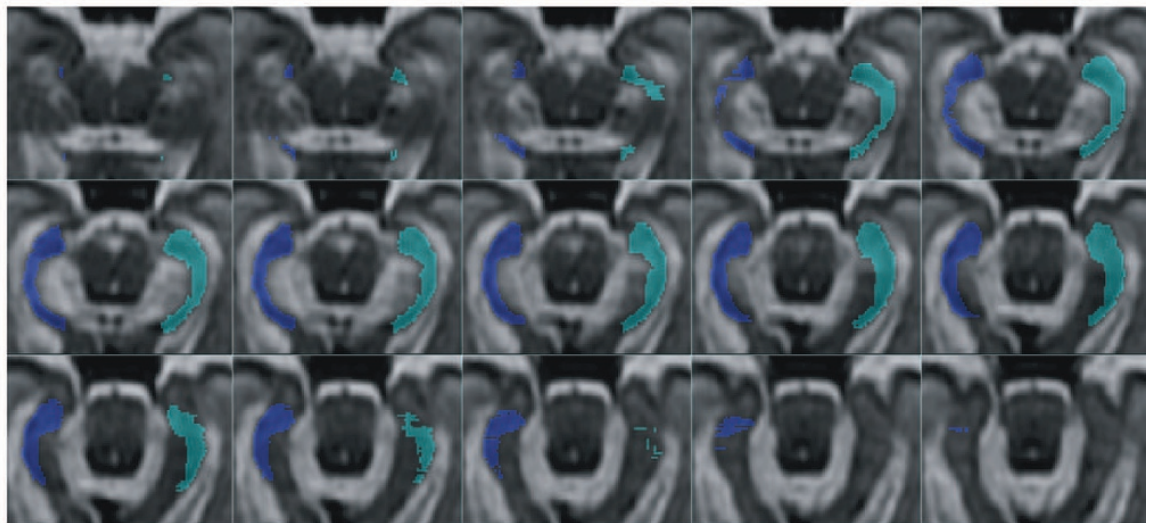

C

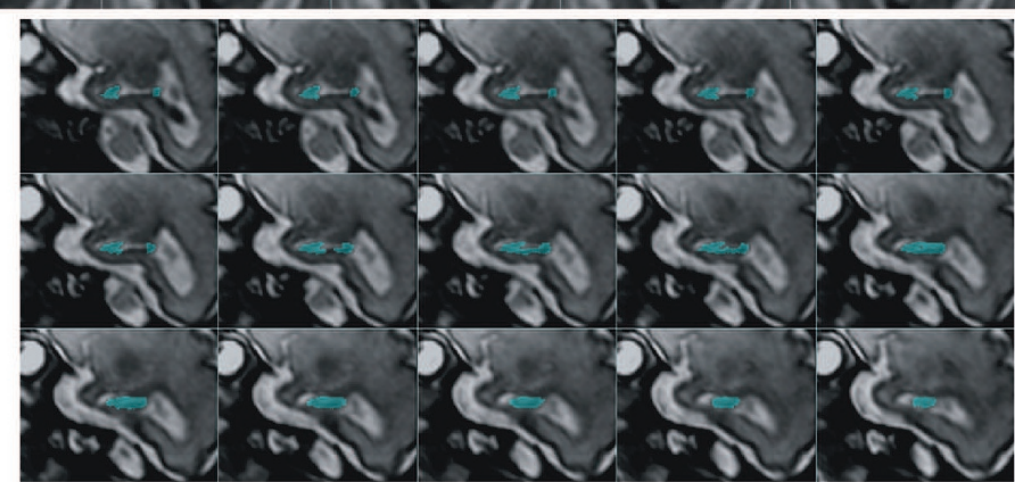

Figure 1. T2-weighted reconstructed images in the $(A)$ coronal plane, $(B)$ axial plane, and $(C)$ sagittal plane representing the segmentation of the right (blue) and left (green) hippocampus of a fetus at 28 wk gestation. $(B)$ and $(C)$ highlight the segmentation of the head, body, and tail of the hippocampal formation, whereas $(A)$ highlights the segmentation of the hippocampal head.

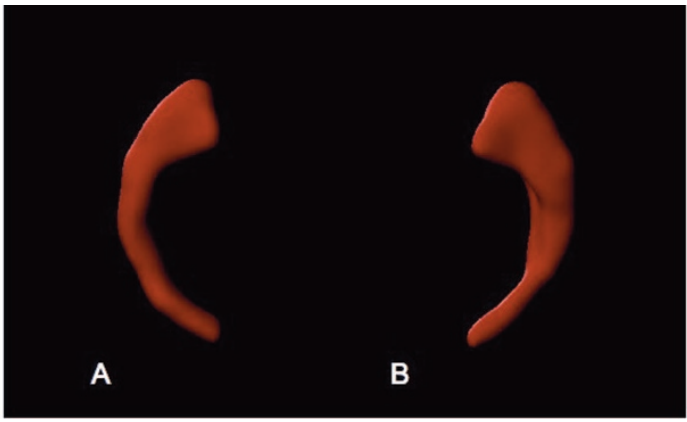

Figure 2. Axial view of a 3D surface model of the right $(A)$ and left $(B)$ hippocampus, based on segmentation of the fetus shown in Fig. 1.

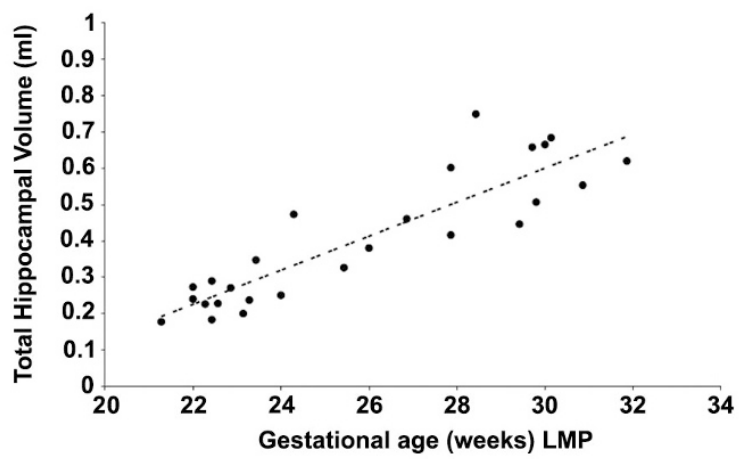

Figure 3. THV as a function of GA. A linear increase in THV can be identified between 21 and 31 gestational wk $\left(p<0.001 ; R^{2}=0.79\right)$ with a calculated equation of THV $(\mathrm{mL})=(0.0468 \times$ gestational weeks $)-0.8034$. 
Table 1. Estimated THV based on GA

\begin{tabular}{cc}
\hline Gestational age $(\mathrm{wk})$ & Average volume $(\mathrm{mL})$ \\
\hline 22 & 0.23 \\
23 & 0.27 \\
24 & 0.32 \\
25 & 0.37 \\
26 & 0.41 \\
27 & 0.46 \\
28 & 0.51 \\
29 & 0.55 \\
30 & 0.60 \\
\hline
\end{tabular}

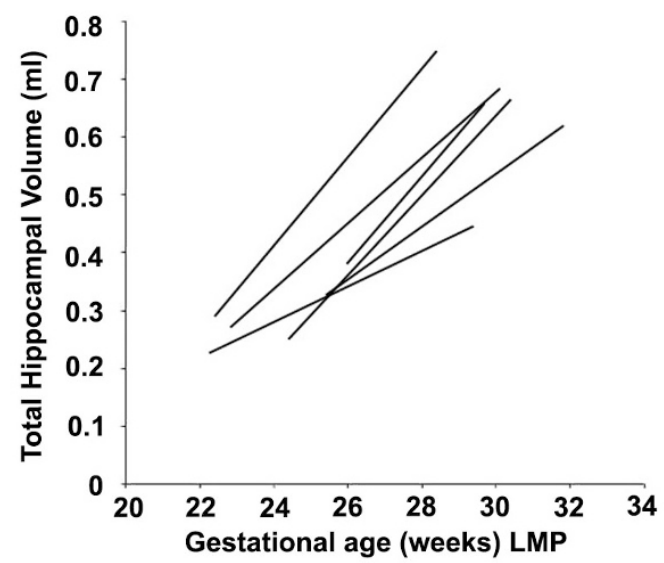

Figure 4. THV as a function of GA for the six subjects who were scanned twice. A linear increase in THV is seen in all subjects scanned twice.

In general, the boundaries of the hippocampus used in our study were most similar to that of Watson et al. (25), with the exception of the posterior boundary. Previous adult and pediatric studies have used the crus of the fornix as the endpoint of hippocampal tail segmentation. This method allows measurement of $90-95 \%$ of the THV (25). However, this structure cannot be consistently identified in fetuses. Therefore, we opted to segment the tail of the hippocampus as far medially as the hippocampal head was segmented, based on the sagittal plane, although this may have resulted in an underestimation of the hippocampal volume.

There have been several studies in children, which have shown a progressive increase in hippocampal volume postnatally $(29,30)$. However, there are no prior volumetric studies of the fetal hippocampus for comparison. Thus, our study provides initial data on fetal hippocampal volume during the second and third trimesters of gestation. Interestingly, if we assume a continued linear increase in THV during the gestational period of 31-40 wk, then THV in our study is lower than hippocampal volumes reported in two neonatal hippocampal volumetric studies $(31,32)$. It is likely that this difference between fetal and neonatal hippocampal volumes is, in part, due to adaptations that were needed to deal with the unique features of the fetal brain and fetal MR images. Thus, the hippocampal volumes in our study were obtained using different methodology. It also possible, however, that the growth of the hippocampus in the late third trimester may be more rapid than the growth that we observed between 21 and 31 gestational wk. Because of this, our results can only be applied to fetuses between 21 and 31 gestational wk and cannot be extrapolated beyond 31 gestational wk. Further studies in older fetuses are needed to determine the growth rate of the hippocampus during later GAs.

In this study, we did not observe a significant difference between THV and sex of the fetus. This is consistent with the findings of Thompson et al. who did not observe a significant difference between sexes in preterm neonates imaged at term. Interestingly, several adult studies have shown that the volume of the hippocampus is larger in men than in women (28), which suggests that these changes occur later. We also did not observe a significant difference between left and right hippocampal volumes, which differs from pediatric studies that have found larger hippocampal volumes on the right as early as term equivalent age (29,32-34). It may be that in the fetal population, the difference in right and left hippocampal volume is too small to be detected with the current technology or that this difference occurs at a later stage of development.

Although, we were able to successfully segment the hippocampus and demonstrate an increase in hippocampal volume with increasing GA, our study has several technical limitations. These include limitations inherent to fetal MRI such as fetal movement, small size of imaged structures, and the large distance between the fetus and the receiver coil (35). Furthermore, we chose to segment the hippocampus based on reconstructed 3D images rather than based on 2D images. Indeed, 2D images are variable in their slice orientation because of the effects of normal fetal motion, and segmentation of structures would be prone to significant errors. Using reconstructed 3D MR images, we were able to reformat the brain in the sagittal, axial, and coronal planes and use these planes to reliably segment the hippocampus in the same manner for all subjects.

Our study was also limited by tissue contrast. At times, segmentation and discrimination of two adjacent structures were limited by contrast resolution. This occurred, for example, when trying to segment the hippocampal formation from the germinal matrix, which is unique to the developing fetal brain. Involution of the germinal matrix begins late in the second trimester and is almost complete by term (36). In the younger fetus, the germinal matrix is present and appears hypointense on T2 images. Below 28 wk gestation, in most cases, it was indistinguishable from the hippocampus on coronal sections; however, it could be easily identified on sagittal sections and by following the wall of the ventricles. However, in certain cases, where differentiation was difficult, segmentation was achieved by changing the intensity of the image, which usually revealed a demarcation between the two structures with the germinal matrix being more hypointense than the hippocampal formation. When any ambiguity still persisted, we chose to include the entire area in the segmentation, which may have resulted in a slight overestimation in some cases. Using a different echo time may better distinguish the boundary of the germinal matrix and adjacent hippocampus depending on the GA; however, we were not able to vary echo times because of the inherent low signal to noise nature of fetal MRI and scan time considerations. 
Although we focused on a limited 10-wk gestational period (between 21 and 31 wk), our study was limited by our small sample size which may have affected our ability to detect differences in hippocampal volume by gender or sidedness. Although right and left hippocampal segmentations were reviewed with a pediatric neuroradiologist in a random order, our ability to detect differences in hippocampal volume by sidedness may have also been limited by the fact that we consistently segmented the right hippocampus first. Additional studies, using a larger number of patients, with random order of hippocampal segmentation is needed to further explore where there are indeed no differences in hippocampal sidedness or gender volume during this gestational period.

In summary, using 3D reconstructed fetal MR images, we were able to adapt previous hippocampal segmentation methodology to segment the fetal hippocampus. We observed a progressive increase in the volume of the hippocampus during the second and third trimesters. This normative data can now be used for comparative studies of conditions suspected to affect in utero hippocampal development, such as intrauterine growth retardation and congenital brain malformations, and can be correlated with neurodevelopmental outcome measures such as learning and memory.

Acknowledgments. We thank Ms. Addison Cuneo for her logistical help, and the volunteers who participated in this study.

\section{REFERENCES}

1. Bohbot VD, Allen JJ, Nadel L 2000 Memory deficits characterized by patterns of lesions to the hippocampus and parahippocampal cortex. Ann N Y Acad Sci 911:355-368

2. Kier EL, Kim JH, Fulbright RK, Bronen RA 1997 Embryology of the human fetal hippocampus: MR imaging, anatomy, and histology. AJNR Am J Neuroradio $18: 525-532$

3. Baker LL, Barkovich AJ 1992 The large temporal horn: MR analysis in developmental brain anomalies versus hydrocephalus. AJNR Am J Neuroradiol 13:115-122

4. Sato N, Hatakeyama S, Shimizu N, Hikima A, Aoki J, Endo K. 2001 MR evaluation of the hippocampus in patients with congenital malformations of the brain. AJNR Am J Neuroradiol 22:389-393

5. Barsi P, Kenéz J, Solymosi D, Kulin A, Halász P, Rásonyi G, Janszky J, Kalóczkai A, Barcs G, Neuwirth M, Paraicz E, Siegler Z, Morvai M, Jerney J, Kassay M, Altmann A. 2000 Hippocampal malrotation with normal corpus callosum: a new entity? Neuroradiology 42(5):339-345

6. Sizonenko SV, Borradori-Tolsa C, Bauthay DM, Lodygensky G, Lazeyras F, Huppi P 2006 Impact of intrauterine growth restriction and glucocorticoids on brain development: insights using advanced magnetic resonance imaging. Mol Cell Endocrinol 254-255:163-171

7. Kuchna I 1994 Quantitative studies of human newborns' hippocampal pyramida cells after perinatal hypoxia. Folia Neuropathol 32:9-16

8. Schmidt-Kastner R, Freund TF 1991 Selective vulnerability of the hippocampus in brain ischemia. Neuroscience 40:599-636

9. Giménez M, Junque C, Narberhaus A, Caldu X, Salgado-Pineda P, Bargallo N, Segarra D, Botet F 2004 Hippocampal gray matter reduction associates with memory deficits in adolescents with history of prematurity. Neuroimage 23:869-877

10. Abernethy LJ, Klafkowski G, Foulder-Hughes L, Cooke RW 2003 Magnetic resonance imaging and T2 relaxometry of cerebral white matter and hippocampus in children born preterm. Pediatr Res 54:868-874
11. Isaacs EB, Lucas A, Chong WK, Wood SJ, Johnson CL, Marshall C, VarghaKhadem F, Gadian DG 2000 Hippocampal volume and everyday memory in children of very low birth weight. Pediatr Res 47:713-720

12. Isaacs EB, Edmonds CJ, Chong WK, Lucas A, Morley R, Gadian DG 2004 Brain morphometry and IQ measurements in preterm children. Brain 127:2595-2607

13. Isaacs EB, Vargha-Khadem F, Watkins KE, Lucas A, Mishkin M, Gadian DG 2003 Developmental amnesia and its relationship to degree of hippocampal atrophy. Proc Natl Acad Sci U S A 100:13060-13063

14. Righini A, Zirpoli S, Parazzini C, Bianchini E, Scifo P, Sala C, Triulzi F 2006 Hippocampal infolding angle changes during brain development assessed by prenatal MR imaging. AJNR Am J Neuroradiol 27:2093-2097

15. Rousseau F, Glenn OA, Iordanova B, Rodriguez-Carranza C, Vigneron D, Barkovich AJ, Studholme C 2005 A novel approach to high resolution fetal brain MR imaging. Med Image Comput Comput Assist Interv 8:548-555

16. Kim K, Hansen MF, Habas PA, Rousseau F, Glenn OA, Barkovich AJ, Studholme C 2008 Intersection-based registration of slice stacks to form 3D images of the human fetal brain. 5th IEEE International Symposium on Biomedical Imaging: From Nano to Macro. Paris, France, pp 1167-1170

17. Kim K, Habas PA, Rousseau F, Glenn OA, Barkovich AJ, Studholme C 2010 Intersection based motion correction of multi-slice MRI for 3D in utero fetal brain image formation. IEEE Trans Med Imaging 29:146-158

18. Habas PA, Kim K, Corbett-Detig JM, Rousseau F, Glenn OA, Barkovich AJ, Studholme C 2010 A spatiotemporal atlas of MR intensity, tissue probability and shape of the fetal brain with application to segmentation. Neuroimage 53:460-470

19. Habas PA, Kim K, Rousseau F, Glenn OA, Barkovich AJ, Studholme C 2010 Atlas-based segmentation of developing tissues in the human brain with quantitative validation in young fetuses. Hum Brain Mapp 31:1348-1358

20. Duvernoy HM 2005 The Human Hippocampus: Functional Anatomy, Vascularization, and Serial Sections With MRI. 3rd ed. Springer, Berlin, pp 5-217

21. Bayer SA, Altman J 2004 Atlas of Human Central Nervous System Development: The Human Brain During the Third Trimester. Vol. 2. CRC Press, Boca Raton, FL, pp 6-197

22. Bayer SA, Altman J 2005 Atlas of Human Central Nervous System Development: The Human Brain During the Second Trimester. Vol. 3. CRC Press, Boca Raton, FL, pp 4-367

23. Humphrey T 1967 The development of the human hippocampal fissure. J Anat 101:655-676

24. Jack CR Jr, Twomey CK, Zinsmeister AR, Sharbrough FW, Petersen RC, Cascino GD 1989 Anterior temporal lobes and hippocampal formations: normative volumetric measurements from MR images in young adults. Radiology 172:549-554

25. Watson C, Andermann F, Gloor P, Jones-Gotman M, Peters T, Evans A, Olivier A, Melanson D, Leroux G 1992 Anatomic basis of amygdaloid and hippocampal volume measurement by magnetic resonance imaging. Neurology 42:1743-1750

26. Jack CR Jr, Bentley MD, Twomey CK, Zinsmeister AR 1990 MR imaging-based volume measurements of the hippocampal formation and anterior temporal lobe: validation studies. Radiology 176:205-209

27. Pantel J, O'Leary DS, Cretsinger K, Bockholt HJ, Keefe H, Magnotta VA, Andreasen NC 2000 A new method for the in vivo volumetric measurement of the human hippocampus with high neuroanatomical accuracy. Hippocampus 10:752-758

28. Geuze E, Vermetten E, Bremner JD 2005 MR-based in vivo hippocampal volumetrics: 1. Review of methodologies currently employed. Mol Psychiatry 10:147-159

29. Pfluger T, Weil S, Weis S, Vollmar C, Heiss D, Egger J, Scheck R, Hahn K 1999 Normative volumetric data of the developing hippocampus in children based on magnetic resonance imaging. Epilepsia 40:414-423

30. Utsunomiya H, Takano K, Okazaki M, Mitsudome A 1999 Development of the temporal lobe in infants and children: analysis by MR-based volumetry. AJNR Am J Neuroradiol 20:717-723

31. Thompson DK, Wood SJ, Doyle LW, Warfield SK, Lodygensky GA, Anderson PJ, Egan GF, Inder TE 2008 Neonate hippocampal volumes: prematurity, perinatal predictors, and 2-year outcome. Ann Neurol 63:642-651

32. Lodygensky GA, Seghier ML, Warfield SK, Tolsa CB, Sizonenko S, Lazeyras F, Huppi PS 2008 Intrauterine growth restriction affects the preterm infant's hippocampus. Pediatr Res 63:438-443

33. Mulani SJ, Kothare SV, Patkar DP 2005 Magnetic resonance volumetric analysis of hippocampi in children in the age group of 6-to-12 years: a pilot study. Neuroradiology 47:552-557

34. Thompson DK, Wood SJ, Doyle LW, Warfield SK, Egan GF, Inder TE 2009 MR-determined hippocampal asymmetry in full-term and preterm neonates. Hippocampus 19:118-123

35. Glenn OA, Barkovich AJ 2006 Magnetic resonance imaging of the fetal brain and spine: an increasingly important tool in prenatal diagnosis, part 1. AJNR Am J Neuroradiol 27:1604-1611

36. Gilles FH, Gomez IG 2005 Developmental neuropathology of the second half of gestation. Early Hum Dev 81:245-253 